\title{
Stokes diagnostics of magneto-convection. Profile shapes and asymmetries.
}

\author{
E.V. Khomenko ${ }^{1,2}$, S. Shelyag ${ }^{3}$, S.K. Solanki ${ }^{3}$, A. Vögler ${ }^{3}$, and \\ M. Schüssler ${ }^{3}$ \\ ${ }^{1}$ Instituto de Astrofísica de Canarias, Tenerife, Spain; \\ ${ }^{2}$ Main Astronomical Observatory, Kyiv, Ukraine email:khomenko@iac.es \\ ${ }^{3}$ Max-Planck-Institut für Aeronomie, Katlenburg-Lindau, Germany \\ email:shelyag@linmpi.mpg.de, solanki@linmpi.mpg.de,voegler@linmpi.mpg.de, \\ msch@linmpi.mpg.de
}

\begin{abstract}
We discuss the polarization signals produced in recent realistic 3D simulations of solar magnetoconvection. The Stokes profiles of the Fe I 6301.5, 6302.5, 15648 and $15652 \AA$ Zeemansensitive spectral lines are synthesised and smeared to simulate the image degradation caused by the Earth's atmosphere and finite telescope resolution. A Principal Component Analysis approach is applied to classify the profiles. We find that the classes of Stokes $V$ profiles as well as their amplitude and area asymmetries are very close to the observations in the network and inter-network regions.
\end{abstract}

We have used 3D MHD simulations produced by the MURaM code (see Vögler et al., 2003). The code includes non-grey radiative transfer, full compressibility and the effects of partial ionization. The snapshot of the simulations used below has a bi-polar structure of the magnetic field with an average unsigned flux of $140 \mathrm{G}$.

The spectra of the FeI 15648, 15652, 6301 and $6302 \AA$ lines have been calculated for every vertical column of the simulation box under the hypothesis of local thermodynamic equilibrium. The Fe abundance used in the line calculations was 7.50 and both micro and macruturbulence were set to zero. In order to provide a comparison of the simulated data with observations, we convolve the Stokes spectra with the point-spread function of the telescope and the atmosphere. The degraded spectra have spatial resolution of 0 " .5 and granulation contrast $\delta I_{c}$ of $7 \%$ in the visible and resolution of $0^{\prime \prime} .8$ and $\delta I_{c}=3 \%$ in the infrared. In addition to smoothing, we averaged 4 by 4 original pixels together, so that the new pixel resolution is equal to $80 \mathrm{~km}$.

Spatial smearing produces an increase of the profile asymmetries. The profiles most frequently obtained in the simulations show positive asymmetry (see Fig. 1). All classes of profiles shown there can be found in observations (c.f. Sánchez Almeida \& Lites, 2000). The irregular shape mixed-polarity profiles make 14\% (Fe I 15648) and 15\% (Fe I 6302) of all the classified profiles (see examples in Fig. 1c, f). In the simulations they are located in between the opposite polarity areas. The profiles of the same class form patches on the surface of $600-700 \mathrm{~km}$ size, close to the spatial resolution.

The asymmetries of the profiles with the degraded resolution are produced by a combination of vertical and horizontal gradients in the $0 . .^{\prime \prime} 5 \times 0 .^{\prime \prime} 5\left(0 .^{\prime \prime} 8 \times 0 . .^{\prime \prime} 8\right.$ for the IR) area. Fig. 2 demonstrates that both the $\delta a$ and $\delta A$ histograms are shifted toward positive values with respect to the case of the original resolution. The STD of the asymmetries are significantly lower in the case of the reduced spatial resolution. The STD of the amplitude asymmetry $\delta a$ in the latter case are similar for the IR and the visible line. 

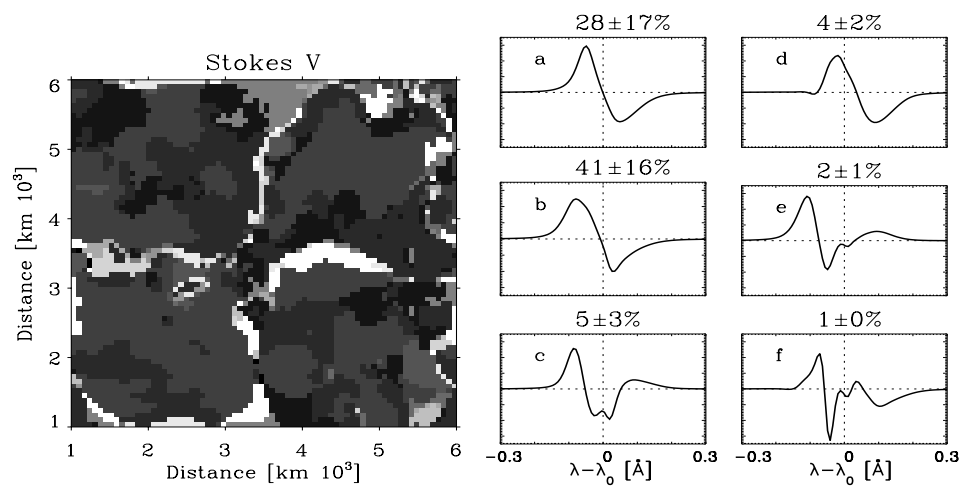

Figure 1. Left panel: map of the classes of the Fe I $6302 \AA$ Stokes $V$ profiles. The grey scaling from black to white corresponds to the change of the zero-crossing shift of the class profiles from red to blue accompanied by the change of the area asymmetry from negative to positive. The locations of the irregular profiles are marked by the lightest colors. Right panel: examples of class profiles. The fraction of line profiles belonging to each class is given in percent at the top of each panel.
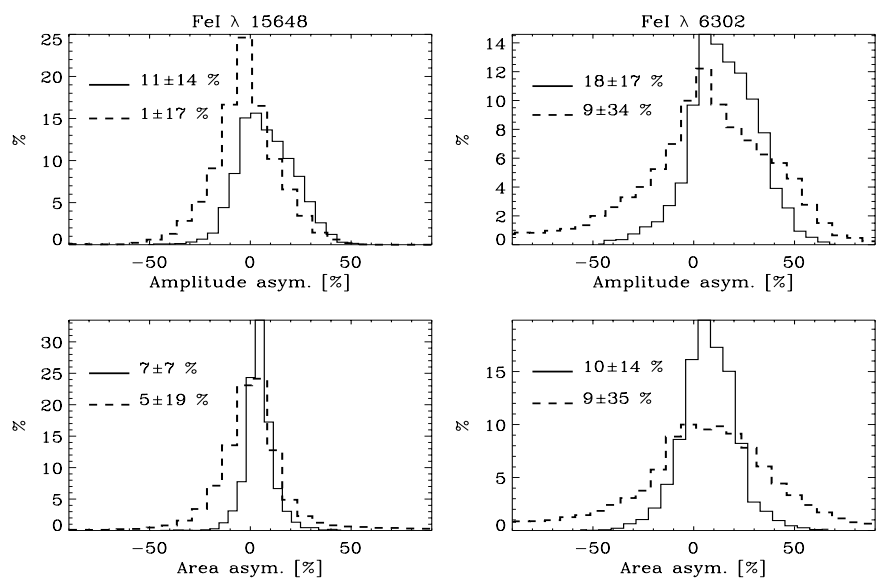

Figure 2. Solid line: Histograms of the amplitude (top) and area (bottom) asymmetries of the Stokes $V$ profiles with the degraded resolution. Dashed line: same for the profiles with the original resolution of $20 \mathrm{~km}$. The mean values and standard deviations corresponding to each curve are given in the figure.

The observations of the quiet Sun (mainly network fields) in the visible lines at $6300 \AA$ give values of $\delta a=15 \%$ and $\delta A=6 \%$ (Sigwarth et al., 1999), while the quiet Sun's inter-network magnetic fields observed at $1.56 \mu \mathrm{m}$ produce profiles with amplitude and area asymmetries of $15 \%$ and $7 \%$, respectively (Khomenko et al., 2003). Thus, the range of asymmetries in the MHD model agrees well with the observational constrains.

\section{References}

Khomenko, E. V., Collados, M., Solanki, S. K., Lagg, A., Trujillo Bueno, J. 2003, A\&A, 408, 1115

Sánchez Almeida, J., Lites, B. W. 2000, ApJ, 532, 1215

Sigwarth, M., Balasubramaniam, K. S., Knölker, M., Schmidt, W. 1999, A\&A, 349, 941

Vögler, A. 2003, Three-dimentional simulations of magneto-convection in the solar photosphere, Ph.D. thesis, Univ. Göttingen 